\title{
Origin of the Thermoreversible fcc-bcc Transition in Block Copolymer Solutions
}

\author{
Timothy P. Lodge and Joona Bang \\ Department of Chemical Engineering \& Materials Science, University of Minnesota, Minneapolis, Minnesota 55455, USA
}

Moon Jeong Park and Kookheon Char

School of Chemical Engineering, Seoul National University, Seoul 151-744, Republic of Korea

(Received 20 June 2003; published 6 April 2004)

\begin{abstract}
The thermoreversible fcc-bcc transition in concentrated block copolymer micellar solutions is shown to be driven by decreases in the aggregation number as the solvent penetrates the core, leading to a softer intermicelle potential. Small-angle neutron scattering measurements in a dilute solution are used to quantify the temperature-dependent micellar characteristics. The observed phase boundary is in excellent agreement with recent simulations of highly branched star polymers.
\end{abstract}

DOI: 10.1103/PhysRevLett.92.145501

Block copolymers are self-assembling soft materials that display a rich variety of order-order and orderdisorder transitions (ODT) [1]. By tuning copolymer molecular weight and composition, solution concentration $(\phi)$, solvent selectivity, monomer incompatibility, and temperature $(T)$, specific transitions can be placed for experimental convenience. Furthermore, the success of modern theory in computing free energies and structural details of the various equilibrium phases makes block copolymers a fertile ground for providing stringent tests of theory. The transition between face-centered (or close-packed) cubic (fcc) and body-centered cubic (bcc) lattices is particularly interesting. The fcc/bcc boundary is widespread in atomic systems, both one component and multicomponent, and is likewise well known in colloidal systems, both charged and uncharged. Consequently, analytical theory and computer simulations have been highly refined [2-7]. This transition has recently been observed in block copolymer micelles [8-13], which are model soft colloids, analogous to highly branched star polymers. In contrast to star polymers, however, the micellar functionality can be tuned with temperature, providing direct access to the thermoreversible fcc/bcc transition.

We have located the fcc/bcc boundary in several polystyrene-polyisoprene (SI) diblock copolymer solutions, in both styrene- and isoprene-selective solvents $[12,13]$. By shear aligning the close-packed phase in small-angle $\mathrm{x}$-ray scattering (SAXS) and small-angle neutron scattering (SANS) cells, we demonstrated that the transition is epitaxial and that the transformation pathway closely follows that seen in atomic systems $[12,14]$. However, beyond speculation about the possible role of changing solvent selectivity, we were not able to pinpoint the precise cause of the transition; why does bcc become favored over fcc upon heating? Here we demonstrate exactly how increasing $T$ affects the micellar characteristics (i.e., aggregation number, core radius, overall radius, solvent concentration in the core, and number density), and compare the results with detailed simula-
PACS numbers: 61.25.Hq, 64.70.Nd, 81.30.Hd, 83.80.Qr

tions of the phase behavior of highly branched star polymers; the agreement between theory and experiment is gratifying.

Two nearly symmetric SI copolymers were synthesized by anionic polymerization [13]. One incorporated perdeuterated styrene $(\mathrm{dS})$, with block molecular weights of 15800 (dS) and 15400 (I), and the other used perdeuterated isoprene (dI), with block molecular weights of $15400(\mathrm{~S})$ and $14100(\mathrm{dI})$. Solutions were prepared with volume concentrations $\phi=0.5 \%, 1 \%$, and $2 \%$ for dSI in tetradecane (C14) and for SdI in diethyl phthalate (DEP). $\mathrm{C} 14$ is isoprene selective, and a mixture of normal and perdeuterated C14 exactly matched the SANS scattering length density of the I corona. Similarly, DEP is styreneselective and almost contrast matches the $\mathrm{S}$ corona. Solutions were measured as a function of $T$ on the $30 \mathrm{~m}$ NG7 spectrometer at the National Institute of Standards and Technology, with wavelength $\lambda=6 \AA$ and $\Delta \lambda / \lambda=$ 0.10 . Raw data were corrected following standard procedures and placed on an absolute intensity scale.

Representative scattering curves are shown in Fig. 1(a) for $0.5 \%, 1 \%$, and $2 \% \mathrm{dSI} / \mathrm{C} 14$ at $30{ }^{\circ} \mathrm{C}$, and in Fig. 1(b) for $1 \% \mathrm{SdI} / \mathrm{DEP}$ solutions as a function of $T$. In Fig. 1(a) the characteristic shape of the hard-sphere form factor is evident, with a distinct minimum near $0.047 \AA^{-1}$, as is the emergence of a structure factor peak at very low $q$ for the highest $\phi$. The main features in Fig. 1(b) are the decreased low $q$ intensity and the smearing of the form factor minimum and secondary maximum with increasing $T$. The data were consistently fit with a detailed model of micellar scattering $[15,16]$, as illustrated by smooth curves. The implemented model includes a spherical micellar form factor, a hard-sphere structure factor, and a Gaussian distribution of core sizes. The fitting parameters most relevant to this discussion are the mean aggregation number $f$, the mean core radius $R_{c}$, and the effective hard-sphere radius $R_{\mathrm{hs}}$. The fits were facilitated by the invisibility of the corona, and the known contrast factors and absolute intensity scale 

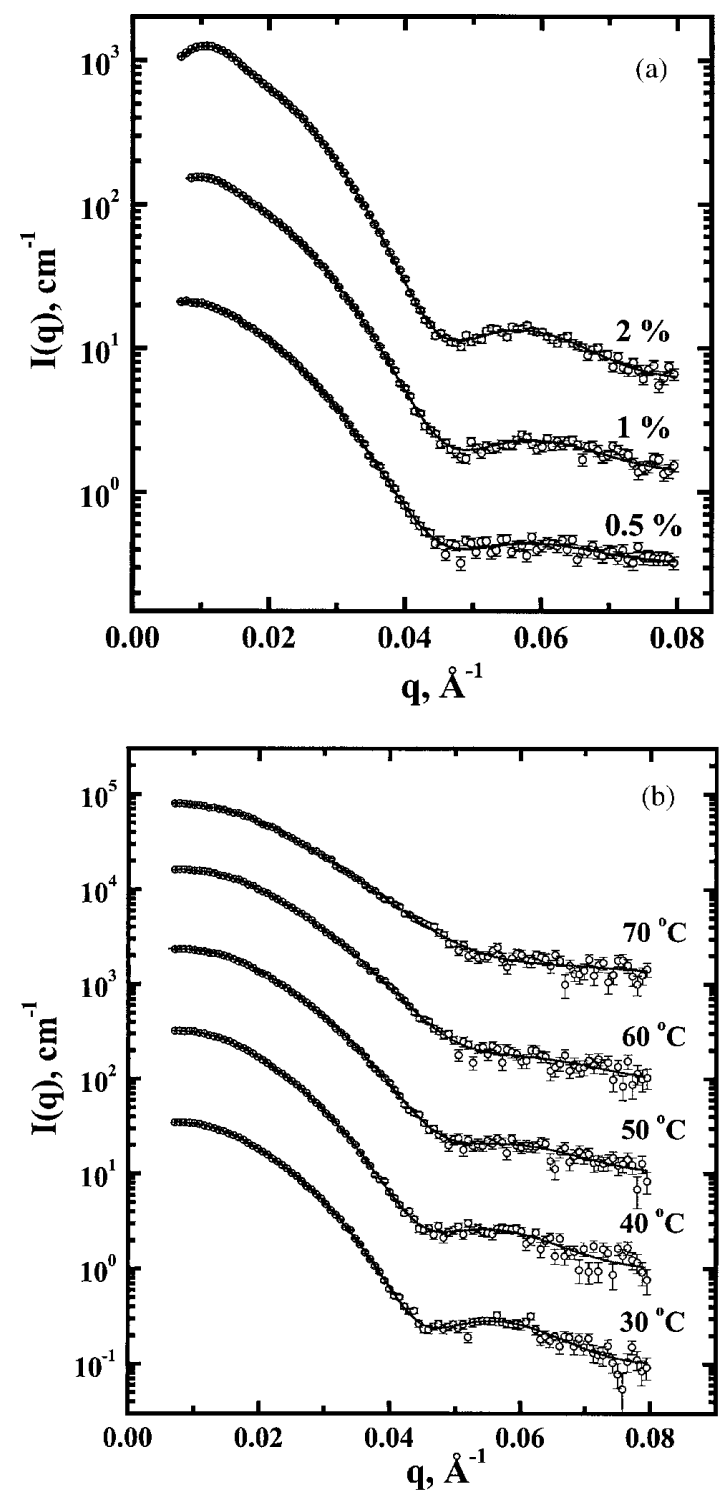

FIG. 1. (a) SANS data (symbols) as function of $\phi$ for dSI in $\mathrm{C} 14$ at $30{ }^{\circ} \mathrm{C}$. For clarity the $1 \%$ and $2 \%$ data have been multiplied by 4 and 16, respectively. The solid lines are the model fits. (b) SANS data as a function of $T$ for $1 \%$ SdI in DEP. For clarity the $40,50,60$, and $70^{\circ} \mathrm{C}$ data have been multiplied by $10,10^{2}, 10^{3}$, and $10^{4}$, respectively. The solid lines are the model fits.

enabled the direct determination of $f$. The solvent fraction in the core, $\phi_{\text {sol, core }}$, was also computed. The resulting micellar parameters were found to be sensibly independent of $\phi$, and data for the $1 \%$ solution are collected in Table I.

These results provide a clear picture of how increasing $T$ modifies the micellar structure. For both systems at low $T$, the cores are essentially solvent-free, and $f$ and $R_{c}$ are constant. As $T$ increases, the solvent begins to penetrate the core appreciably. This is accompanied by a decrease in $f, R_{c}$, and $R_{\mathrm{hs}}$, as the lower interfacial tension at the core/ corona interface allows the stretched core blocks to relax.
The micelles disappear at the critical micelle temperature (CMT) (ca. 90 and $80{ }^{\circ} \mathrm{C}$ for dSI/C14 and SdI/DEP, respectively), when the solvent quality toward the core block has improved sufficiently for dispersed single chains to be favored. Dynamic light scattering was also used to determine the hydrodynamic radius of the micelles, $R_{h}$, and $R_{h}$ matched $R_{\mathrm{hs}}$ very well in terms of both magnitude and $T$ dependence.

SAXS measurements were also made on a $15 \%$ solution of dSI in C14, which shows the fcc/bcc boundary at $66^{\circ} \mathrm{C}$ and the ODT at $92^{\circ} \mathrm{C}$, and a $25 \%$ solution of SdI in DEP, with the fcc/bcc boundary at $70^{\circ} \mathrm{C}$ and the ODT at $84^{\circ} \mathrm{C}$. Measurements were taken on the University of Minnesota 2 m SAXS line, as previously described $[13,14]$. The $T$ dependence of the principal peak position, $q^{*}(T)$, is shown in Fig. 2. This quantity shows a $T$ independent regime at low $T$, and then a smooth increase with increasing $T$; in particular, $q^{*}(T)$ varies smoothly across the fcc/ bcc transition. If we make the reasonable assumption that the micelles are just touching on their respective lattice, a "nearest-neighbor" radius $R_{\mathrm{nn}}$ can be computed from $q^{*}$ using the appropriate lattice parameter $a_{\mathrm{fcc}}$ or $a_{\mathrm{bcc}}$ as

$$
R_{\mathrm{nn}}=\frac{\sqrt{6}}{4} \frac{2 \pi}{q^{*}}=\left\{\frac{\sqrt{2}}{4} a_{\mathrm{fcc}} \text { or } \frac{\sqrt{3}}{4} a_{\mathrm{bcc}}\right\} .
$$

Similarly, the number density of micelles, $n / V$, can be computed as $4 / a_{\mathrm{fcc}}{ }^{3}$ or $2 / a_{\mathrm{bcc}}{ }^{3}$, respectively. These quantities are also gathered in Table I. The notable features are that $n / V$ is constant at low $T$ but begins to increase as $T$ increases, and that $R_{\mathrm{nn}}$ follows the same trends as $R_{c}$ and $R_{\mathrm{hs}}$ in dilute solution.

In order to relate the micellar characteristics at low $\phi$ to the ordered solutions at higher $\phi$, we postulate an analog to the well-known Natta-Corradini rules for polymer crystallization, namely, that the crystal unit cell is determined first by the minimization of intramolecular conformational energetics, followed by optimization of the interchain packing [17]. In this case, we propose that the micellar structure is optimized as a function of $T$ but does not depend much on $\phi$, and thus that the dilute solution results are directly applicable to the ordered state. (Note that this hypothesis cannot be applied to arbitrarily high $\phi$, where the samples transform into hexagonal cylinder phases [13].) There are five reasons behind this postulate. First, the low $T$ regime of constant dimensions (dilute micelles) and constant $q^{*}$ (concentrated micelles) match well. Second, in the concentrated solutions the low $T$ values of $f$, inferred from $n / V$ and the known $\phi$, agree well with the dilute case. Third, the low $\phi$ CMT is virtually identical to the higher $\phi$ ODT, indicating that the latter is dictated by solvent quality. The correspondence of the dilute solution CMT and the concentrated solution ODT as a general phenomenon in block copolymer solutions was noted previously [13]. Fourth, the $T$ dependences of $R_{\mathrm{hs}}$ and $R_{\mathrm{nn}}$ (and $R_{h}$ ) track 
TABLE I. Micellar Characteristics

\begin{tabular}{|c|c|c|c|c|c|c|c|c|c|c|}
\hline \multicolumn{11}{|l|}{ dSI in C14 } \\
\hline \multirow[b]{2}{*}{$T\left({ }^{\circ} \mathrm{C}\right)$} & \multicolumn{4}{|c|}{ SANS ( $1 \%$ solution) } & \multicolumn{4}{|c|}{ SAXS (15\% solution) } & \multirow[b]{2}{*}{$\%$ free chains } & \multirow[b]{2}{*}{ phase } \\
\hline & $R_{c}(\AA)$ & $R_{\mathrm{hs}}(\AA)$ & $f$ & $\phi_{\text {core,sol }}{ }^{a}$ & $q^{*}\left(\AA^{-1}\right)$ & $R_{\mathrm{nn}}(\AA)$ & $a(\AA)^{\mathrm{b}}$ & $n / V 10^{16} \mathrm{~cm}^{-3}$ & & \\
\hline 30 & 98 & 230 & 161 & 0 & 0.0169 & 228 & 646 & 1.5 & 0.0 & fcc \\
\hline 40 & 99 & 230 & 168 & 0 & 0.0169 & 228 & 646 & 1.5 & 0.0 & fcc \\
\hline 50 & 94 & 216 & 134 & 0.02 & 0.0169 & 228 & 646 & 1.5 & 17 & $\mathrm{fcc}$ \\
\hline 60 & 88 & 197 & 94 & 0.15 & 0.0178 & 216 & 612 & 1.7 & 31 & fcc \\
\hline 70 & 83 & 187 & 77 & 0.19 & 0.0198 & 194 & 448 & 2.2 & 28 & bcc \\
\hline 80 & 79 & 186 & 63 & 0.23 & 0.0215 & 179 & 414 & 2.8 & 25 & bcc \\
\hline
\end{tabular}

SdI in DEP

\begin{tabular}{|c|c|c|c|c|c|c|c|c|c|c|}
\hline \multirow[b]{2}{*}{$T\left({ }^{\circ} \mathrm{C}\right)$} & \multicolumn{4}{|c|}{ SANS ( $1 \%$ solution) } & \multicolumn{4}{|c|}{ SAXS ( $25 \%$ solution) } & \multirow[b]{2}{*}{$\%$ free chains } & \multirow[b]{2}{*}{ phase } \\
\hline & $R_{c}(\AA)$ & $R_{\mathrm{hs}}(\AA)$ & $f$ & $\phi_{\text {core,sol }}{ }^{\mathrm{a}}$ & $q^{*}\left(\AA^{-1}\right)$ & $R_{\mathrm{nn}}(\AA)$ & $a(\AA)^{\mathrm{b}}$ & $n / V 10^{16} \mathrm{~cm}^{-3}$ & & \\
\hline 30 & 103 & 187 & 184 & 0 & 0.0219 & 176 & 497 & 3.3 & 0.0 & fcc \\
\hline 40 & 103 & 182 & 160 & 0.10 & 0.0219 & 176 & 497 & 3.3 & 13 & fcc \\
\hline 50 & 97 & 170 & 121 & 0.17 & 0.0219 & 176 & 497 & 3.3 & 34 & fcc \\
\hline 60 & 93 & 164 & 99 & 0.24 & 0.0233 & 165 & 468 & 3.9 & 36 & fcc \\
\hline 70 & 86 & 151 & 67 & 0.34 & 0.0245 & 157 & 363 & 4.2 & 53 & bcc \\
\hline
\end{tabular}

${ }^{\mathrm{a}} \phi_{\text {core, sol }}$ is the volume fraction of solvent in the core. $4 / 3 \pi R_{c}{ }^{3}=f V_{\text {core-block }} /\left(1-\phi_{\text {core,sol }}\right)$

${ }^{\mathrm{b}} a$ is either $a_{\mathrm{fcc}}$ or $a_{\mathrm{bcc}}$ depending on the structure.

each other closely. Fifth, previous workers established the validity of this approach in aqueous polyethyleneoxidepolybutyleneoxide (PEO-PBO) systems [11,18].

This postulate allows us to conclude that the fcc to bcc transition is driven primarily by decreasing $f$. Decreasing $f$ dilutes the corona brush, and thereby softens the intermicellar potential. We can compare these data with a recent theory for highly branched star polymers. The key assumption in so doing is that so long as the coronas do not overlap very much, micelles will be equivalent to highly branched stars; they both can be considered as spherically symmetric particles coated with tethered chains. Of course, micelles can adjust their aggregation

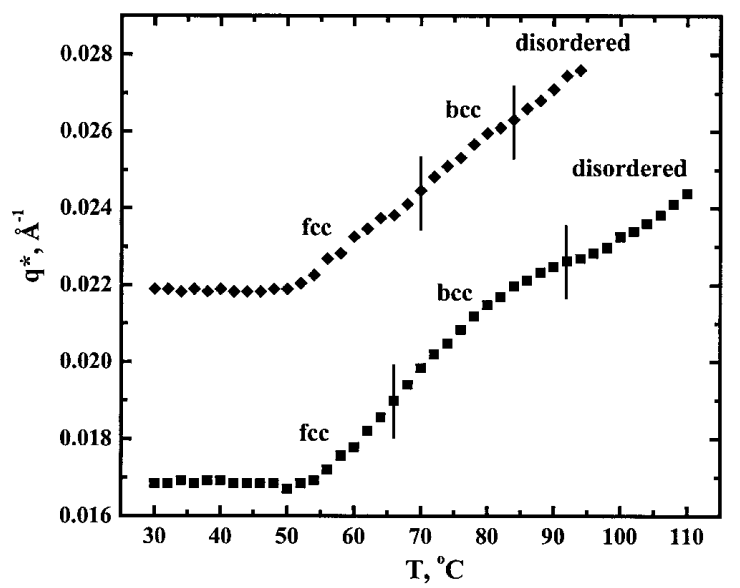

FIG. 2. The principal peak position $q^{*}$ measured by SAXS as a function of $T$ for dSI in C14 $\phi=15 \%$ (squares) and SdI in DEP $\phi=25 \%$ (diamonds). number in response to changes in $T$, as exemplified here, and also in response to increased packing at still higher $\phi$, and so the analogy must be used with caution. Watzlawek et al. used a two-part pair potential consisting of a logarithmic core inside a Yukawalike exponential tail to simulate the phase behavior [5]. Figure 3 shows the relevant low $\phi$ portion of their phase boundary, as a plot of $1 / f$ versus packing fraction $\eta$. Our data are indicated as a series of points; the agreement is qualitatively excellent, and quantitatively encouraging. The packing fraction of micelles is estimated as follows:

$$
\eta=\frac{\pi}{6} \frac{n}{V} \sigma^{3} \approx \frac{\pi}{6} \frac{n}{V}\left(\frac{2 R_{\mathrm{nn}}}{1+2 / \sqrt{f}}\right)^{3}
$$

where $\sigma$ is the boundary distance in the two-part potential [5]. This quantity is mapped onto the diameter of the micelle corona up to the center of the outermost "DaoudCotton" blob $[19,20]$, i.e., the range of facile overlap of neighboring coronas is restricted to half of the outermost blob of the starlike particle. As noted by Watzlawek et al. [5], decreasing $f$ in the simulation can be viewed as increasing $T$. The "critical value" of $f$ for the transition is approximately $80 \pm 10$ in both solutions, whereas in the simulation it is closer to 60-70. The experimental results also indicate a significant and increasing fraction of free chains with increasing $T$. This quantity, also listed in Table I, may be estimated based on the assumption that all the chains are in micelles at low $T$. Stiakakis et al. [21] have shown that the presence of free chains softens the interstar (or intermicelle) potential, which would have the effect of stabilizing the bcc phase. Thus accounting 


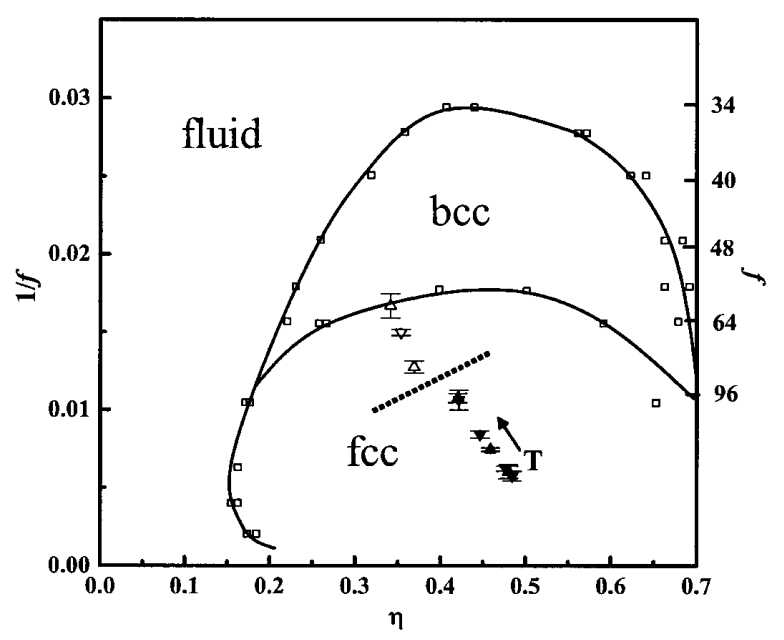

FIG. 3. The phase diagram of star polymer solutions for different arm numbers $f$ versus packing fraction $\eta$, reproduced from Ref. [5]. Up and down triangles represent dSI in C14 and SdI in DEP, respectively, and the solid and open symbols indicate fcc and bcc phases, respectively. The dotted line is the guideline for the fcc/bcc transition in this study.

for the presence of free chains might even bring the theory and experiment into perfect agreement.

These results may also be compared with the pioneering work of McConnell and Gast, who reported either fcc or bcc phases of SI diblocks of different composition in decane $[8,9]$. They found bcc packing for "hairy" micelles and fcc for "crew-cut" micelles, as the intermicelle potential varied from long range to more hard- spherelike. This is qualitatively consistent with the explanation here, in that decreasing $f$ induces a softer repulsion between micelles. However, these authors did not vary $T$, and thus the thermotropic fcc/bcc phase boundary was not located [9]. We note that Hamley and co-workers described thermoreversible fcc/bcc transitions in amphiphilic PEO-PBO block copolymers [10]. In their case, fcc was obtained upon heating, as the solvent quality of water for the PEO corona blocks decreased, leading to a shrinking of the corona into a denser object. This phenomenon therefore has an important qualitative difference from our system, in which the corona block is always under good solvent conditions. Recently Castelletto et al. have examined in detail the $T$ dependent characteristics of dilute PEO-PBO micelles by SANS [16]. In agreement with our results, the solvent fraction in the core decreased and the aggregation number increased with diminished solvent quality, but the fits also quantified the substantial corona shrinkage that is not echoed in our system. Also, the sample examined adopted the bcc lattice exclusively at higher $\phi$, and so comparisons of micellar characteristics and the location of the fcc/bcc boundary could not be made.
In summary, we have shown that temperature-induced changes in micellar characteristics, particularly the mean aggregation number, can be quantified in dilute solution, and that this information can be combined with data for higher concentration, ordered micellar solutions to understand the observed fcc/bcc transition. The results demonstrate that the softening of the intermicelle potential with decreasing aggregation number is the key feature, in nearly quantitative agreement with recent simulations of star polymers. Block copolymer micelles are appealing as model soft colloids, as temperature offers a direct route to tuning the pair potential, in terms of both range and steepness.

This work was supported primarily by the MRSEC Program of the National Science Foundation under Grant No. DMR-0212302, and also in part by the Brain Korea 21 Program endorsed by the Korean Ministry of Education. We acknowledge helpful discussions with C. N. Likos.

[1] I.W. Hamley, The Physics of Block Copolymers (Oxford University Press, Oxford, 1998).

[2] W. G. Hoover, D. A. Young, and R. Grover, J. Chem. Phys. 56, 2007 (1972).

[3] B. B. Laird and A. Haymet, Mol. Phys. 75, 71 (1992).

[4] M. O. Robbins, K. Kramer, and G. S. Grest, J. Chem. Phys. 88, 3286 (1988).

[5] M. Watzlawek, C. N. Likos, and H. Löwen, Phys. Rev. Lett. 82, 5289 (1999).

[6] B. Groh and M. Schmidt, J. Chem. Phys. 114, 5450 (2001).

[7] P. Ziherl and R. D. Kamien, J. Phys. Chem. B 105, 10147 (2001).

[8] G. A. McConnell et al., Phys. Rev. Lett. 71, 2102 (1993).

[9] G. A. McConnell and A. P. Gast, Macromolecules 30, 435 (1997).

[10] I.W. Hamley, J. A. Pople, and O. Diat, Colloid Polym. Sci. 276, 446 (1998).

[11] I.W. Hamley et al., Langmuir 16, 2508 (2000).

[12] J. Bang et al., Phys. Rev. Lett. 89, 215505 (2002).

[13] T. P. Lodge, B. Pudil, and K. J. Hanley, Macromolecules 35, 4707 (2002).

[14] J. Bang and T. P. Lodge, J. Phys. Chem. B 107, 12071 (2003).

[15] J. S. Pedersen and M. C. Gerstenberg, Macromolecules 29, 1363 (1996).

[16] V. Castelletto, I.W. Hamley, and J. S. Pedersen, J. Chem. Phys. 117, 8124 (2002).

[17] G. Natta and P. Corradini, J. Polym. Sci. 39, 29 (1959).

[18] N. Deng et al., J. Polym. Sci. 33, 1085 (1995).

[19] C. N. Likos and H. M. Harreis, Condens. Matter Phys. 1, 173 (2002).

[20] M. Daoud and J. P. Cotton, J. Phys. (Paris) 43, 531 (1982).

[21] E. Stiakakis et al., Phys. Rev. Lett. 89, 208302 (2002). 